\title{
6G: Opening New Horizons for Integration of Comfort, Security and Intelligence
}

Guan Gui, Senior Member, IEEE, Miao Liu, Member, IEEE, Fengxiao Tang, Member, IEEE, Nei Kato, Fellow, IEEE, and Fumiyuki Adachi, Life Fellow, IEEE

The innovations of sixth generation wireless communication $(6 \mathrm{G})$ as compared to fifth generation $(5 \mathrm{G})$ are considered in this paper based on the analysis of related works. With the aim of improving multiple communication targets in each service, five $6 \mathrm{G}$ core services are identified for different target requirements. Two centricities and eight key performance indices (KPIs) are detailed to describe these services, and enabling technologies are discussed to satisfy the KPIs. A 6G architecture is proposed as an integrated system of the enabling technologies, and is then illustrated using four typical application scenarios. Potential challenges for development of 6G technology is then discussed and possible solutions are proposed. Finally, opportunities for exploring $6 \mathrm{G}$ are analyzed in order to guide future research.

\section{RELATED WORKS}

Commercial approvals have been officially declared for the deployment of fifth generation (5G) wireless systems in numerous countries, with $5 \mathrm{G}$ enabled smart phones and infrastructures already appearing in the market. However, prior to the recently emerging commercial applications of $5 \mathrm{G}$, research exploring future wireless systems has already extended to the concept of beyond 5G (B5G). Furthermore, since 2018, scholars have begun to focus on the concept of $6 \mathrm{G}$ and its applications [1]. B.

G. Gui and M. Liu are with the College of Telecommunications and Information Engineering, Nanjing University of Posts and Telecommunications, Nanjing 210003, China (e-mails: \{guiguan,liumiao\}@njupt.edu.cn)

F. Tang and N. Kato are with the Graduate School of Information Sciences, Tohoku University, Sendai 980-8577, Japan (e-mails: \{fengxiao.tang, kato\}@it.is.tohoku.ac.jp)

F. Adachi Wireless Signal Processing Research Group, Research Organization of Electrical Communication (ROEC), Tohoku University, Sendai, Miyagi, 980-8577 Japan (e-mail: adachi@ecei.tohoku.ac.jp) 
Zong et al. have explored the key drivers and new logical start of 6G [2], while Z. Zhang et al. also shared their viewpoints on $6 \mathrm{G}$ and detailed a number of promising techniques, such as terahertz (THz) communications, large intelligent surfaces (LISs), orbital angular momentum (OAM), blockchain-based spectrum sharing, quantum communications and computing, molecular communications [3]. R. Heath described three key directions for $6 \mathrm{G}$ in the physical layer [4], while P. Yang et al. detailed several advanced techniques of $6 \mathrm{G}$ in the transmission and network layer [5]. Additionally, K. Letaief et al. provided a development roadmap of $6 \mathrm{G}$ with artificial intelligence (AI) based technologies [6]. In [7] and [8], the trends were further extended for $6 \mathrm{G}$ were discussed. To the extent of the authors' knowledge, a lack of comprehensive consideration of the $6 \mathrm{G}$ roadmap remains, including its relationships with $\mathrm{B} 5 \mathrm{G}$, core services, distinctive targets, required KPIs, enabling technologies, architecture, potential challenges, promising solutions, opportunities, and the future developmental direction towards of 6G.

\section{CORE SERVICES FOR 6G}

\section{A. Distinction: $5 G, B 5 G$, and $6 G$}

To provide a foundation for the discussion of $6 \mathrm{G}$ services, $5 \mathrm{G}$ core services of enhanced mobile broadband (eMBB), ultra-reliable low latency communications (URLLC), and massive machine type communications (mMTC) are first reviewed. The research targets are improved data rate, transmission latency, and connectivity performance, which address the most urgent wireless communication issues for present demands. Numerous studies have explored the effects of various $5 \mathrm{G}$ technologies such as orthogonal frequency division multiple access (OFDMA), polar code, massive multiple input multiple output (MIMO), millimeter wave (mmWave), and software defined network (SDN) on the defined three targets. Various additional techniques for improving the performance targets of 5G include full duplex, non-orthogonal multiple access (NOMA), and mobile edge computing (MEC). From this perspective, B5G can be considered as an evolution to further enhance $5 \mathrm{G}$ performance.

As demands for service quality accelerate, an increasingly number of wireless services must be optimized for further targets. New radio lite (NR-Lite) for $5 \mathrm{G}$ has been proposed by the third Generation Partnership Project (3GPP) for Release 17 [9]. The NR-Lite complements eMBB, URLLC and mMTC to achieve the targets of improved data rate, transmission latency, and connectivity performance. However, NR-Lite is only able to achieve performance tradeoffs among these targets, and struggles to improve them simultaneously. Hence, NR-Lite can only be applied to low tier users with multiple 
medium requirements and cannot satisfy the emerging demands of users with multiple high performance requirements. To support higher demands for $6 \mathrm{G}$ users on multiple performance targets, considering $6 \mathrm{G}$ with multiple performance improvements rather than tradeoffs has become a popular perspective $[1][3][8]$.

\section{B. Evolution: scenario centric and comfort based $6 G$}

Four kinds of $6 \mathrm{G}$ core services are identified for enhanced performance combined with $5 \mathrm{G}$. These include enhanced eMBB + URLLC, enhanced eMBB + mMTC, enhanced URLLC + mMTC, and tradeoff based enhanced eMBB + URLLC + mMTC. In this article, the services are denoted as MBBLL (mobile broad bandwidth and low latency), mBBMT (massive broad bandwidth machine type), mLLMT (massive low latency machine type) and 6G-Lite. Furthermore, to prove the rationality of the evolution of $6 \mathrm{G}$ core services, potential killer applications for the $6 \mathrm{G}$ services are predicted as follows.

Typical applications of MBBLL include mobile augmented reality (AR), virtual reality (VR), and holographic teleconferencing [2]. Such applications require high data rates (downlink peak rate $>1$ Tbps, user experienced rate $>10 \mathrm{Gbps}$ [3]) for high definition video streams and large amounts of interactive instructions, as well as low latencies for real-time voices $(<0.1 \mathrm{~ms})$ and immediate control responses $(<1 \mathrm{~ms})$ [7]. These requirements must also be guaranteed in high-mobility scenarios $(>1000 \mathrm{~km} / \mathrm{h} \mathrm{[3])} \mathrm{for}$ future use in space exploration, traveling over the air and sea, and maglev transportation.

For mBBMT, the tactile Internet of Things (IoT) will be an important use scenario by the 2030s [3], demanding higher data rates to support touch related experiences. The IoT also requires massive connectivity for the densely deployed (e.g., $100 / \mathrm{m}^{3}$ ) sensors and devices to capture tactile sensations and transform them into digital data. Subsequently, mLLMT will attract a large amount of attention for use in the large scale industrial IoT (IIoT) with applications including smart manufacturing and automatic transportation. These applications will require massive connectivity for communications among staff, sensors, and actuators, but also low latency to deal with the frequent interactions between these devices.

Considering the complex diversification and personalization requirements with multiple social and individual orientations, intelligent driving will provide the best scenario for 6G-Lite. In intelligent driving scenarios, multiple events must be jointly considered, including path planning, automatic driving, obstacle detection, vehicle monitoring, mobile entertainment, and emergency rescue operations. High rate, low latency, and massive connectivity must all be achieved under high-mobility conditions to support the $6 \mathrm{G}$ applications. However, considering the current capabilities of 5G NR-Lite, it is impossible 
to satisfy all three performance requirements to the maximum extent. Thus, tradeoffs must be optimized for 6G-Lite among different performance targets for various events.

In summary, the evolutionary services detailed above aim to improve joint QoEs of human and machines based on multiple targets and events in complex physical scenarios. They can be collectively referred to as scenario centric services in the physical world with a target of providing users with high quality wireless experiences.

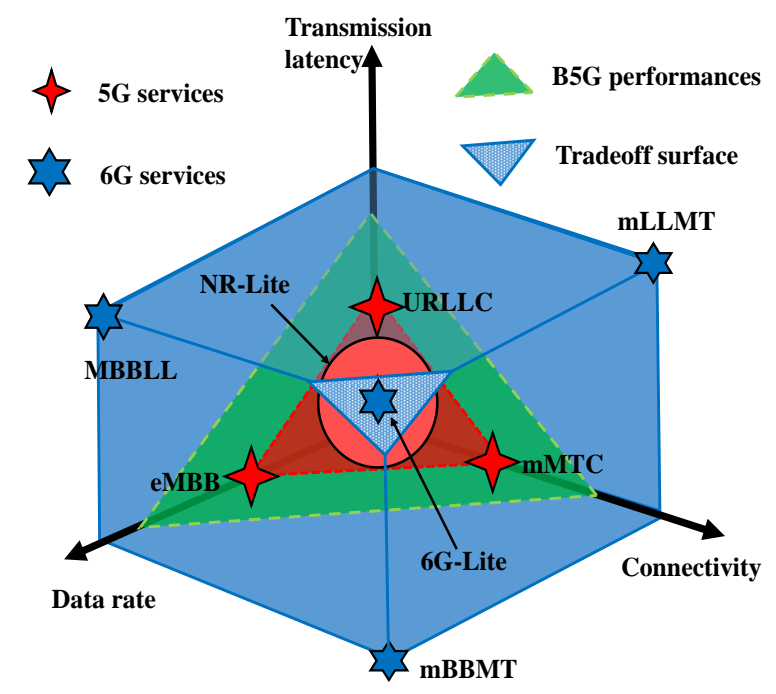

Fig. 1 Evolution from 5G and B5G to 6G.

\section{Revolution: computing centric and security based $6 G$ digital service}

Contrasting to the centric services based on $5 \mathrm{G}$ and B5G evolutions, the fifth $6 \mathrm{G}$ core service is focused on providing secure wireless computing in the digital world. With the development of data mining, cloud-edge computing, mobile caching, and deep learning (DL), general computing has become an important service objective of wireless communications. However, with the utilization of network function visualization and network slicing, wireless systems are scheduled more flexibly. Therefore, wireless computing is also essential for networks to self-optimize transceiver schemes globally. This kind of service is applied to computing data non-locally and for transmitting results, without specific requirement to achieve concrete experiences in actual events and scenarios. Thus, data rate, latency, and connectivity are not the main KPIs of computing and this must be considered as a new service class with corresponding performance targets in the $6 \mathrm{G}$ system.

In addition, cyber and physical layer security issues within wireless networks are widespread in daily 
life. As a result, for wireless computing for individuals and communities, privacy leakage is a predominant concern caused by perpetual data uploading, caching, and transmitting. Providing wireless computing with trusted communications is a crucial requirement. Hence, security should be considered as a performance target of wireless computing in $6 \mathrm{G}$, and is referred to as secure wireless computing for private data (SWCPD).

By combining the evolutionary services and revolutionary services detailed above, a comprehensive vision of $6 \mathrm{G}$ core services can be identified. The services not only provide comfortable experiences to human and machines, but also guarantee security for general computing. To intelligently support comfortable and secure 6G services, KPIs are introduced next, as illustrated in Fig. 2. As shown in the figure, KPIs include both distinctive KPIs for specific services, and common KPIs for general services. Enabling technologies to improve KPIs are discussed in the following section.

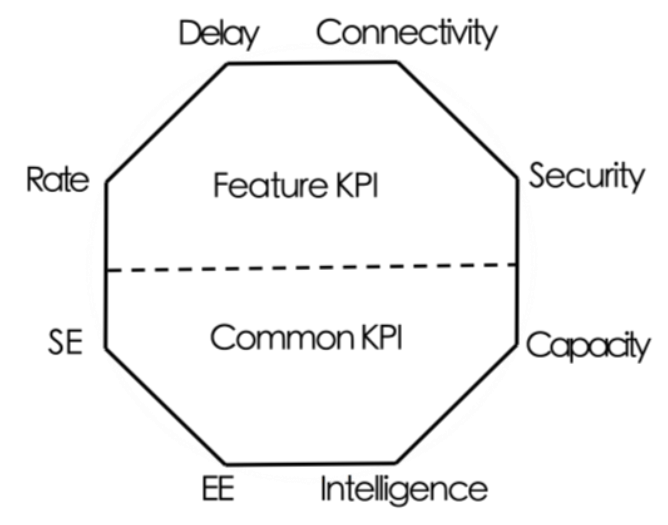

Fig. 2. KPIs for $6 \mathrm{G}$ services.

\section{ENABLING TECHNOLOGIES FOR 6G}

\section{A. For improving data rate}

Advanced modulation schemes are required to improve the data rate for users. The most promising schemes are index modulation and spatial modulation, which utilize the indices of resource blocks and antennas to deliver extra bits. As no additional resources are required, the corresponding spectrum efficiency (SE) and energy efficiency (EE) can also be improved. Assigning more bandwidth is a direct method to improve data rate. As the spectrum of present frequency band is increasingly scarce, developing new frequency bands higher than extremely high frequency (EHF) band, such as submmWave, terahertz, and visible light is a critical requirement.

\section{B. For reducing latency and guaranteeing reliability}


An emerging technique for reducing latency is the DL based transmission prediction. Data driven DL systems can proactively predict the user's requests and time-varying channel states, leading to shorter transmission delays. To reduce operation delays, model driven DL can be used to train deep neural networks (DNNs) and replace traditional algorithms with an online accelerated DNNs. Distributed and cooperative processing are also important methods to reduce operation delays based on local and cooperative operations on edge devices. Obstacles cause an additional problem for reliability. As frequency becomes higher, obstacles between transceivers block the signals more frequently. To improve reliability, configurable meta-surface can control the propagation environment during communications.

\section{For increasing connectivity}

Connective range and density can be improved to increase the connectivity of the system. For the aspect of range, the most promising technique is the space-air-ground-sea integrated (SAGSI) network. Additional media based networks must also be integrated into 6G systems, including sonar based underwater networks, magnetic induction based underground networks, molecule based Nano-IoT, and the physiological tissue based body access network. Three main directions require exploration to address density issues. Firstly, compressed sensing and sparse coding can reduce the communication data volume for users by compressing information. Edge caching and social network can then proactively store popular content in partial communities, thus reducing centric rate demands. Similarly, transfer learning can reduce data volume for computing services through shared DNN structures. Optical fibers are also an efficient way to reduce the wireless rate demands in backhaul networks and extremely dense wireless networks (eDWNs). Improving SE and capacity can also address density problems are discussed in latter subsections.

\section{For enlarging system capacity}

According to Shannon's theory, the system capacity can be improved through enhancing bandwidth, signal-to-interference plus noise ratio (SINR), and SE. Utilizing EHF is the most direct method to improve capacity, while channel enhancement and interference-noise management are important techniques to address increasing SINR. Channel state can be improved through applying meta-material based configurable intelligent antennas and surfaces to optimize the beams and the propagation channels. Additionally, unmanned aerial vehicle (UAV), balloon, and satellite based A2G communications are also able to improve channel conditions due to line of signs (LoS) with few obstacles and paths.

\section{E. For enlarging $S E$}


As mentioned above, index and spatial modulations are effective physical layer methods to enlarge SE. As EHF and eDWN are promising for use in the 6G system, super massive MIMO can also be considered to enhance the system SE further. On the link layer, novel multiple access methods can accommodate more links on the same spectrum. Among these advanced multiple access methods, NOMA, and rate splitting multiple access (RSMA) are based on non-orthogonal spectrum access, while (OAM) mode assignment provides the foundation for utilizing OAM-based vortex waves. More flexible spectrum management and sharing schemes can also be adopted among heterogeneous services and users to replace traditional schemes like cognitive radio networks, LTE in unlicensed spectrum (LTE-U), and full duplex. In addition to individual network solutions, eDWN and the SAGSI network can further improve the spatial three-dimensional (3D) SE through reusing frequency more densely in adjacent networks.

\section{F. For enhancing EE}

Index and spatial modulations have been noted as effective physical layer methods to enhance EE. Wireless power transferring and simultaneous wireless information and power transferring are also promising technologies for supplying energy to wireless receivers based on energy harvesting. Utilizing interference power as energy source to harvest is a particularly notable potential method. Additionally, low-resolution multiple antenna architectures for MIMO systems are effective for reducing power consumption and raising EE. Distributed and cooperative multiple point transmissions can then effectively reduce power consumption for dense cell based communications, while relay and multi-hop transmissions can lower power consumption for long distance communications at the cost of higher complexities and latencies.

\section{G. For promoting security}

Multiple novel schemes have been considered to manage different kinds of threats in order to improve data privacy and communication security for $6 \mathrm{G}$ services, especially computer centric threats. Secure channel coding, channel based adaptation, artificial interfering signals, and secret sequence extraction are the principal physical layer techniques based on classical information theory [10]. Based on the nocloning theorem and entanglement nature in quantum physics, information can be safely and securely transmitted with classical bits and channels using quantum key distribution and quantum teleportation, respectively. According to the trends of computing centric services and $6 \mathrm{C}$ convergence, cyber-attacks will present another critical problem for $6 \mathrm{G}$ security. For detecting malicious activities, DL based attack 
prediction methods are promising for such a constantly changing environment [11].

\section{H. For realizing intelligence}

The significance of DL in 6G systems is illustrated by the various enabling technologies detailed above. Wireless communication systems have become increasingly intelligent at all levels, assisted by DL. Popular earlier techniques on the physical layer include data-driven DL methods for automatic modulation classification, channel state estimation and prediction, adaptive encoding and decoding, intelligent beamforming, and constellation design. For the data link layer, deep reinforcement learning based resource allocation has been widely researched, and for the transportation layer, intelligent traffic prediction and route computing have been proposed to replace inflexible protocols. To reduce the caching and computing costs of learning, lightweight DNNs and distributed learning has been subsequently applied in wireless networks.

\section{ARCHITECTURE FOR 6G: CONNECTION AND INTEGRATION}

To provide comfortable, secure, and intelligent 6G services, enabling technologies must be integrated as an organic whole. For this purpose, a novel $6 \mathrm{G}$ architecture is illustrated in Fig. 3. Methods to achieve technical integrations to support $6 \mathrm{G}$ requirements are then discussed, with four typical applications provided within the architecture.

\section{A. Network coverage integration}

To connect services anywhere, the different coverage of the networks must be integrated. Space, air, and remote areas can be connected into the $6 \mathrm{G}$ core network using satellites. To improve the performance of these long range communications that are far away from the ground, relays can be deployed on balloons, UAVs, ships, and buoys. Alternatively, in urban areas, users can be served by macro base station (BS), micro BS, and simple access point (AP) directly, or relayed by UAVs and other users.

\section{B. Network type integration}

As there are no integrated frameworks for heterogeneous networks (HetNets), jointly considering users and services in the same scenarios but in different type of networks is challenging. To connect everything under the integrated $6 \mathrm{G}$ coverage, the different types of the networks must also be integrated in both aspects of protocol and organization. Particularly in the development of $6 \mathrm{G}$ technologies, it is vital to integrate current HetNets first, including macrocell, femtocell, WiFi, WSN, IoT, and IoV, with centralized, distributed, and cooperative organization. 


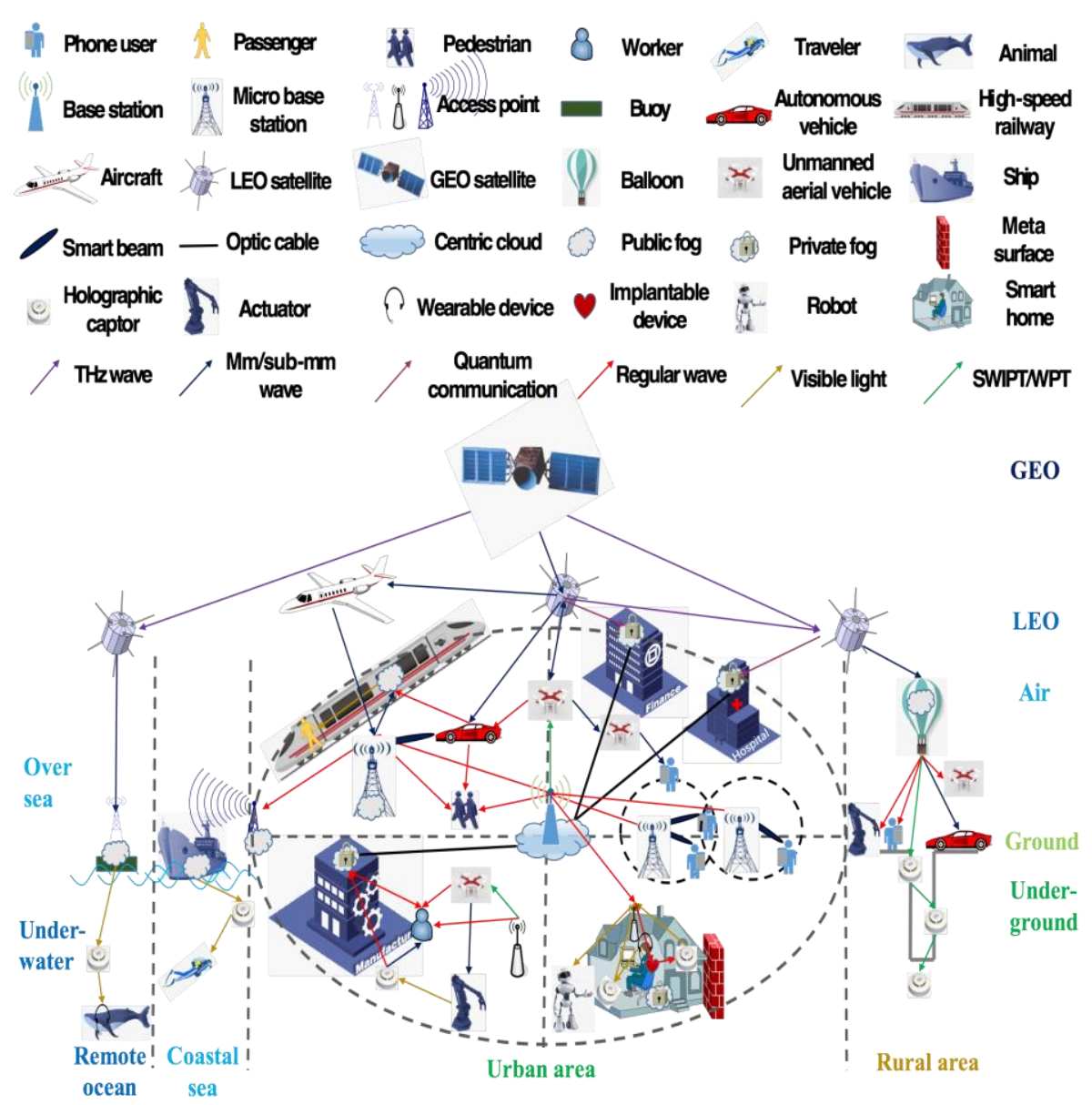

Fig. 3. Proposed $6 \mathrm{G}$ architecture.

\section{Wireless spectrum integration}

Different types of networks will always work on the licensed and unlicensed frequencies exclusively, leading to inefficient spectrum utilization. Employing visible light and EHF requires new scheduling policies. Thus, all available spectrums must be integrated and managed flexibly with higher SE to facilitate the integration of different networks.

\section{Communication medium integration}

Radio signals cannot be propagated everywhere and will not work in areas including underwater, underground, and in the human body. As varying networks may require different media, additional media must also be integrated into the $6 \mathrm{G}$ framework, including optical fiber for backhaul networks, magnetic induction for underground networks, sonar for underwater networks, physiological tissue for body access network, and molecular for Nano-IoT. 


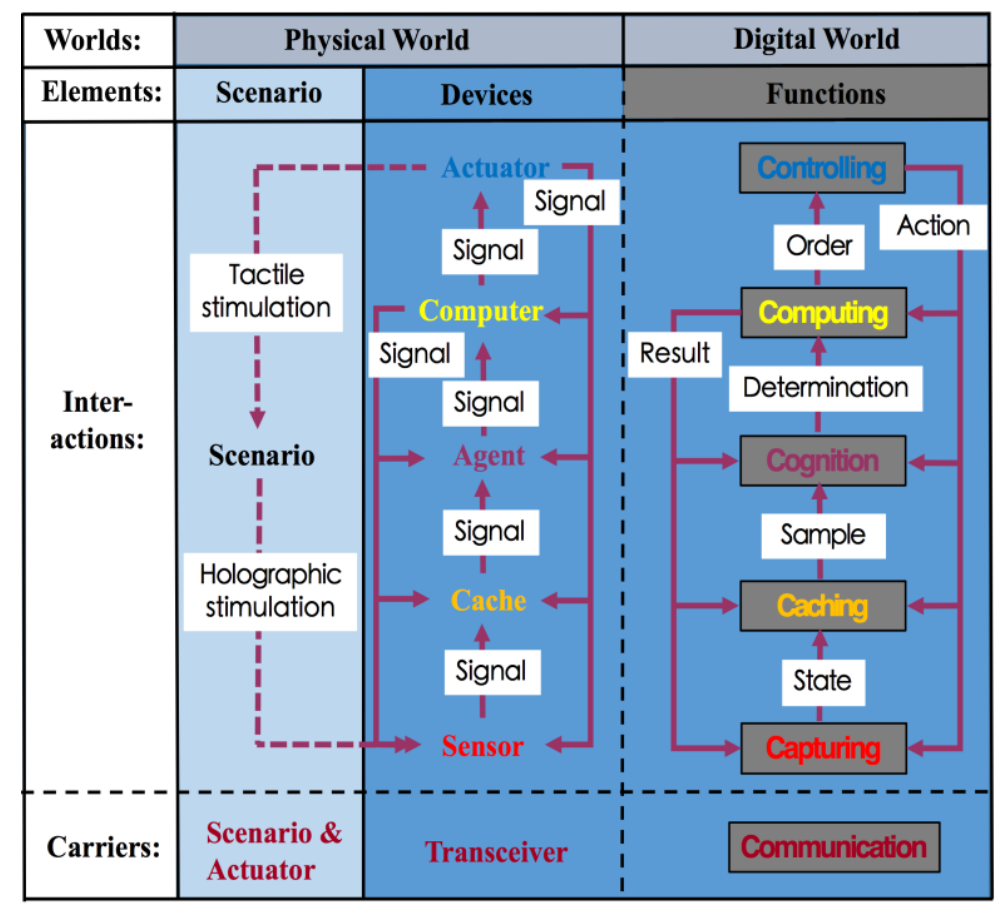

Fig. 4. Convergent loop for 6C.

\section{E. Interactive function integration}

To achieve these targets, six fundamental functions of capturing, communication, caching, cognition, computing, and controlling (6C) are essential. Capturing generally denotes high level sensing, and it is critical for acquiring holographic stimulations from the scenarios. The captured states are then transformed into digital data and cached locally or transformed into physical signals and transmitted to the other devices for further processing. Before processing the data, cognition should be applied to formulate feasible determinations according to the input samples, then computing can accomplish the tasks securely, based on intelligent determinations. Finally, the computing results can be fed back to the former stages for further computer centric utilization. Alternatively, the computed orders will be sent to the actuators for controlling the scenarios and the devices with tactile stimulations and wireless signals. The convergent loops of $6 \mathrm{C}$ interacting with the scenario in the physical and the digital worlds are depicted in Fig. 4. As illustrated, by considering the integrations for the functions, the targets of comfort and security can be improved globally by intelligence, leading to improved experiences for humans and machines in the same scenario.

\section{F. Core service integration}

It can be observed from Fig. 4 that it is necessary to provide both computing and scenario centric services for $6 \mathrm{C}$ interactions. Scenario centric services produce large amounts of data for wireless 
computing and analyzing, while computing centric services feedback computed results to improve the performance of the scenario centric ones. In particular, intelligence is a common technology to improve the performance of both scenario and computing centric services. Therefore, scenario and computing centric services will also be considered as integrations for globally improving system performance and user experience. This integrated service relationship is illustrated in Fig. 5.

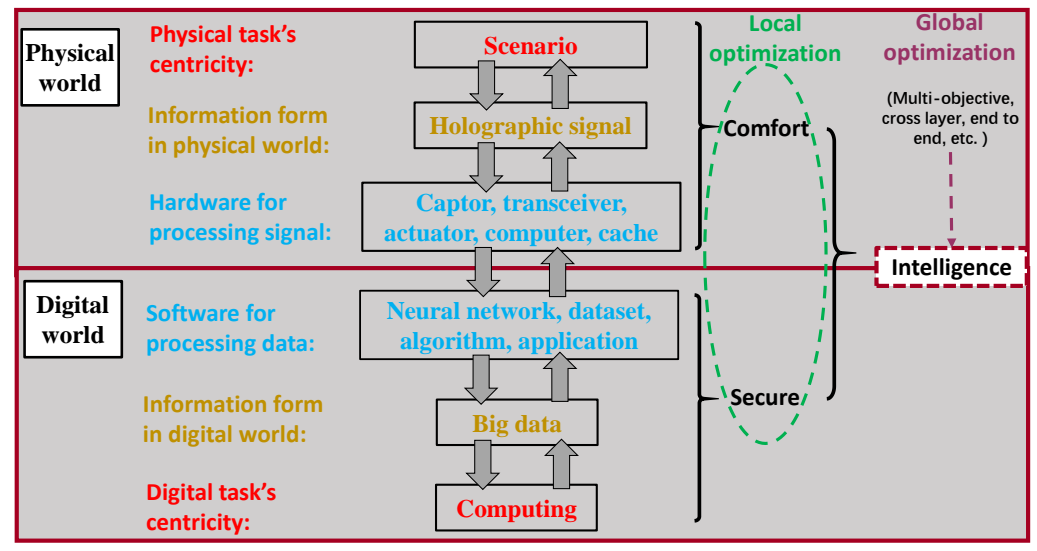

Fig. 5. Integrated scenario centric and computing centric services for global optimization.

\section{G. Layer Integration}

It is necessary to improve individual service KPIs globally in addition to multiple-service integration. Based on multiple-layer based communication models (i.e., open system interconnection (OSI) and transmission control protocol/internet protocol (TCP/IP) reference models), traditional schemes are always considered for optimizing KPIs on a single layer. Previous research into communications with DL have also encountered this issue. As a result, it is necessary to integrate the layers and jointly optimize the KPIs for each service.

\section{ChallengeS AND Possible SOLUTIONS}

The new proposals also present a series of additional problems. In this section, foreseeable challenges for $6 \mathrm{G}$ implementation are discussed and possible solutions are presented.

\section{A. Peak data rate}

$6 \mathrm{G}$ radio must be designed to utilize EHF to increase the peak data rate. It is also critical to build EHF mobile channel models according to scenarios and flexible spectrum management methods must also be considered. Another more serious concern is how to guarantee accurate beam arrivals in highmobility scenarios. Signals on EHF bands experience large path losses and the communication range is 
very short. Thus, a massive number of APs must be deployed, leading to eDWN. With the deployment of eDWNs, frequent handoffs will be another problem in mobile scenarios along with the economic drawbacks of continuously deploying eDWNs over entire service areas.

Potential solutions to these issues are introduced. Firstly, DL is employed as a powerful tool for designing hardware and software. The benefits of DL managing different resources flexibly has been witnessed through the digitalization and visualization trends of the communication system. Outdoor positioning technology with centimeter level accuracy is then introduced for achieving accurate beam arrivals. In addition, macro BS, micro BS, and multi-AP based cooperative transmission are possible solutions to reduce the frequency of handoffs.

\section{B. Latency and reliability}

Although cooperative processing can reduce latency, it raises the cost of communications for link negotiating and data sharing, and increases communication overheads and operation delays. Similarly, current DL based predictions rely on big data, which also introduces more overheads for data collection. Most DL schemes must train DNNs offline. Thus, if the channel state, network topology, and user policy change, the DNN must be retrained, leading to delays for online applications. Hierarchical execution of tasks is an excellent solution for reducing the overheads and delays caused by cooperation. Cross layer optimization and end-to-end based DL schemes are also helpful to optimize the transceiver and propagation environment as a whole to control an environment with meta-surfaces.

\section{Connectivity}

There are numerous challenges involved in integrating networks with different media to increase the quality of connectivity. These include both hardware issues of device compatibility and software issues of system generality. Digitalization, visualization, and DL are possible answers. To increase the density of connectivity, the main challenge is to improve the positioning accuracy, particularly in outdoor and mobile situations, as nearly 100 devices should be connected in the space of only $1 \mathrm{~m}^{3}$. Thus, advanced positioning technology with centimeter level accuracy can be utilized for managing densely deployed devices.

\section{System capacity}

The challenges of enlarging capacity lie in optimizing channel states for A2G networks and taking advantage of interference power. Deep reinforcement learning and intelligent surface are proposed to address these issues as technologies based on intelligent policy and environment optimizations, 
respectively. Global power, subchannel, and beam optimization are also crucial, and can jointly control the interference in the system.

E. SE

Dense reuse of the same frequency to enhance SE will introduce additional interference. In addition, sm-MIMO has high requirements for beam optimization, channel modeling, and transceiver design. Challenges for OAM include the limited number of available OAM modes, joint OAM mode, and frequency/time partition and channel estimation for different OAM modes [12]. Common and critical challenges for flexible spectrum sharing among users and systems is also interference utilization. Detailed methods to solve OAM related challenges have been provided in [12]. For additional challenges, deep reinforcement learning, intelligent surface, and global power, subchannel, and beam optimization schemes are also potential solutions.

F. $E E$

The main challenges when applying the technologies to raising EE lie in the hardware design. It is first necessary to improve harvesting efficiencies of WPT and SWIPT. With the utilization of EHF, new hardware must then be developed for energy harvesting. It is also difficult to design feasible antenna architectures for complicated environments. To deal with the hardware designing difficulties, DL, function digitalization, and configurable meta-materials have been recently proposed as solutions for improving the EE significantly.

\section{G. Promote security}

Numerous issues remain in classical physical layer security, DL based cyber security, and quantum aided communication security. As the challenges and potential solutions have been summarized in [10], [11] and [13], they are not explored again in this work. The necessity of jointly considering the physical layer and cyber security with quantum methods for $6 \mathrm{G}$ services is emphasized here however, particularly for those related to computing and DL. With the popularity and necessity of data collecting for learning in computing centric services, designing a joint secure framework will be the main challenge for promoting total security of the $6 \mathrm{G}$ system. Federated learning is a possible solution based on distributed learning and transferring learning, meaning that all user data is saved locally, and only abstracted models will be shared. Endogenous security has also been put forward as a way to assist systems to establish protection strategies proactively and automatically through joint consideration of physical layer-cyber securities and classical-quantum channels. 


\section{H. Network intelligence}

The current DNNs are almost black box and heavily rely on training datasets. Developed DNN models for wireless communication are also limited [14] [15], and are mainly borrowed from the fields of computer vision. As a result, DL based wireless communication networks only focus on simple tasks with a single target. The majority of schemes also require offline training with a large data set and lack of generalization ability, limiting the capabilities of instant deployment and dynamic self-adaption. The existing intelligent communication researches have only aimed at optimization of the performance of $5 \mathrm{G}$ and $\mathrm{B} 5 \mathrm{G}$ schemes.

For $6 \mathrm{G}$, the systems and the schemes must be originally produced by cognition, instead of optimization. Additionally, existing DL and deep reinforcement learning methods rely heavily on formulated datasets and spaces of states and policies, and cannot operate in unpredictable situations. Current DL methods also only teach agents the best policies under different determined conditions, thus it is difficult to carry out tradeoffs among various targets in dynamic and complicated $6 \mathrm{G}$ environments. To solve the explainability and generalization problems, models based on DL schemes such as deep unfolding neural networks are potential choices, and should be developed based on strict mathematical deductions. Module based DNNs, such as deep unfolding neural networks, also provide better explainability for utilization, while transfer learning can improve the generalization capability. In addition, DL cooperating with analysis of game theory could possibly achieve tradeoff policy among various targets for multiple agents.

\section{OPPORTUNITIES AND FUTURE RESEARCH TRENDS}

Promising opportunities for $6 \mathrm{G}$ are discussed based on related science and technology. According to the enabling technologies and the possible solutions, AI will significantly improve the system performance of 6G. Blockchain is another potentially useful method to manage the 6G systems flexibly and securely, while quantum computing will also provide increased computing efficiency. Furthermore, future developments in mathematics, biology, psychology, sociology, and energy and material sciences will also provide possible inspirations for progress in $6 \mathrm{G}$.

Some future research trends are briefly introduced. Applying meta-learning, lightweight NN, graph $\mathrm{NN}$, capsule $\mathrm{NN}$, and $\mathrm{NN}$ architecture searching on intelligent $6 \mathrm{G}$ research is of significance for $6 \mathrm{G}$. Based on blockchain, all available resources including spectrums and data can then be stored and shared 
through distributed blockchain transactions and protected by consensus protocols and cryptographic security, with no need of entrusting any central party for the ledger maintenance. Quantum computing can also be used to accelerate the speed of information processing, leading to more optimal solutions for 6G communications with shorter time. Furthermore, future research will not only rely on optimization algorithm, compressive sensing, and game theory for digital processing, but will require technologies of biology, energy, and material sciences to develop friendly devices for humans and the environment, aiming at health protection, convenient operation, service life extension, pollution prevention and energy consumption reduction. Meanwhile, psychology and sociology are essential for improved understanding of individual user and societal requirements.

\section{CONCLUSION}

Future 6G scenarios have been explored in this article, including the core services, use cases, KPIs, enabling technologies, architectures, typical scenarios, existing challenges, possible solutions, opportunities, and research directions. As this paper is based upon the authors' previous investigations, imagination, and analysis, the aim is to provide inspiration for future research into $6 \mathrm{G}$.

\section{REFERENCES}

[1] K. David and H. Berndt, “6G vision and requirements,” IEEE Veh. Technol. Mag., vol. 13, no. 9, pp. 72-80, 2018.

[2] B. Zong, C. Fan, X. Wang, X. Duan, B. Wang, and J. Wang, "6G technologies: Key drivers, core requirements, system architectures, and enabling technologies," IEEE Veh. Technol. Mag., vol. 14, no. 3, pp. 18-27, 2019.

[3] Z. Zhang et al., "6G wireless networks: Vision, requirements, architecture, and key technologies," IEEE Veh. Technol. Mag., vol. 14, no. 3, pp. 28-41, 2019.

[4] R. W. Heath, “Going toward 6G,” IEEE Signal Process. Mag., vol. 36, no. 3, pp. 3-4, 2019.

[5] P. Yang, Y. Xiao, M. Xiao, and S. Li, "6G wireless communications: Vision and potential techniques,” IEEE Netw., vol. 33, no. 4, pp. 70-75, 2019.

[6] K. B. Letaief, W. Chen, Y. Shi, J. Zhang, and Y. A. Zhang, "The roadmap to 6G - AI empowered wireless networks," IEEE Commun. Mag., vol. 57, no. 8, pp. 84-90, 2019.

[7] F. Tariq, M. R. A. Khandaker, S. Member, and K. Wong, “A speculative study on 6G," avaliable online: https://arxiv.org/abs/1902.06700

W. Saad, M. Bennis, and M. Chen, "A vision of 6G wireless systems: Applications, trends, technologies, and open research problems," IEEE Network, to be published, doi: 10.1109/MNET.001.1900287

[9] Qualcomm, “NR-Lite for Rel-17 Qualcomm views,” Newport Beach, 2019.

[10] J. M. Hamamreh, H. M. Furqan, and H. Arslan, "Classifications and applications of physical layer security techniques 
for confidentiality : A comprehensive survey,” IEEE Commun. Surv. Tutorials, vol. 21, no. 2, pp. 1773-1828, 2019.

[11] M. Husák, J. Komárková, E. Bou-harb, and P. Celeda, "Survey of attack projection, prediction, and forecasting in cyber security,” IEEE Commun. Surv. Tutorials, vol. 21, no. 1, pp. 640-660, 2019.

[12] W. Cheng, H. Zhang, L. Liang, H. Jing, and Z. Li, “Orbital-angular-momentum embedded massive MIMO: Achieving multiplicative spectrum-efficiency for mmWave communications," IEEE Access, vol. 6, pp. 2732-2745, 2018.

[13] L. Gyongyosi, S. Imre, S. Member, and H. V. Nguyen, “A survey on quantum channel capacities,” IEEE Commun. Surv. Tutorials, vol. 20, no. 2, pp. 1149-1205, 2018.

[14] G. Gui, H. Huang, Y. Song, and H. Sari, “Deep learning for an effective nonorthogonal multiple access scheme," IEEE Trans. Veh. Technol., vol. 67, no. 9, pp. 8440-8450, Sept. 2018.

[15] Nei Kato, Zubair Md. Fadlullah, Bomin Mao, Fengxiao Tang, Osamu Akashi, Takeru Inoue, Kimihiro Mizutani, "The deep learning vision for heterogeneous network traffic control: Proposal, challenges, and future perspective," IEEE Wirel. Commun., vol. 24, no.3, pp. 146-153, 2017. 